\title{
On characterization for a class of pseudo-convex domains with positive constant pseudo-scalar curvature on their boundaries
}

\author{
SONG-YING LI
}

\begin{abstract}
For any compact strictly pseudoconvex pseudo-Hermitian CR manifold $\left(M, \theta_{0}\right)$ in the sense of Webster, the solution of CR-Yamabe problem concludes that there is a contact form $\theta$ which conformally equivalent to $\theta_{0}$ so that the psudo scalar curvature for $(M, \theta)$ is a constant. The current article gives a natural and easily verified sufficient condition, and proves that if $(M, \theta)$ satisfies the condition and has a positive constant pseudo scalar curvature on $M$, then $(M, \theta)$ must be $\mathrm{CR}$ equivalent to the unit sphere.
\end{abstract}

\section{Introduction and main results}

Let $(M, \theta)$ be a strictly pseudo-convex pseudo-Hermitian compact hypersurface in $\mathbb{C}^{n+1}$ in the sense of Webster [36] with a pseudo-Hermitian real one-form $\theta$ on $M$. Let $R_{\theta}$ be the Webster pseudo-scalar curvature for $M$ with respect to $\theta$. By the solution of the CR Yamabe problem given by Jerison and Lee [18], Gamara and Yacoub [10] and Gamara [9] (for $n=1$ ), there is a pseudo-Hermitian real one-form $\theta$ so that $(M, \theta)$ has constant Webster pseudo-scalar curvature $R_{\theta}$. Let $\rho$ be a defining function for $M$. Then $\theta=\frac{1}{2 i}(\partial \rho-\bar{\partial} \rho)$ is a pseudo-Hermitian one-form for $M$, and any Hermitian one-form can be constructed in this way by using defining function of $M$. When $M=S^{2 n+1}$, the unit sphere in $\mathbb{C}^{n+1}$, if $\rho(z)=|z|^{2}-1$, then $R_{\theta}=n(n+1)$ on $M$. The main purpose of the paper is to give some characterizations on $\rho$ so that the pseudo-scalar curvature $R_{\theta}$ is a positive constant on $M$ if and only if $M$ is CR-equivalent to the sphere $S^{2 n+1}$.

Let $D$ be a smoothly bounded pseudo-convex domain in $\mathbb{C}^{n}$. Let $u$ be a strictly plurisubharmonic exhaustion function for $D(u=+\infty$ on $\partial D)$. Let $\rho(z)=-e^{-u(z)}$. Then the Fefferman's functional $J(\rho)$ of $\rho$ is defined as:

$$
J(\rho)=-\operatorname{det}\left[\begin{array}{cc}
\rho & \bar{\partial} \rho \\
(\bar{\partial} \rho)^{*} & H(\rho)
\end{array}\right],
$$


where $H(\rho)$ is the complex Hessian matrix of $\rho, \bar{\partial} \rho=\left(\partial_{\overline{1}} \rho, \ldots, \partial_{\bar{n}} \rho\right)$ is a $1 \times n$ matrix and $(\bar{\partial} \rho)^{*}$ is its adjoint matrix.

Let $f(z)$ be a positive function on $D$. It was known (see, for example, $[7,25])$ that

$$
J(\rho)=f(z)>0 \text { in } D ; \quad \rho=0 \text { on } \partial D
$$

if and only if

$$
\operatorname{det} H(u)(z)=f(z) e^{(n+1) u} \text { in } D ; \quad u=+\infty \text { on } \partial D .
$$

When $f(z) \in C^{\infty}(\bar{D})$ is positive for all $z \in \bar{D}$, the existence and uniqueness of a strictly plurisubharmonic solution of (1.3) were given by Cheng and Yau in [7]. In particular, when $f(z) \equiv 1$, they proved that the plurisubharmonic solution $u$ of (1.3) defines a complete Kähler-Einstein metric on $D: \frac{\partial^{2} u}{\partial z_{i} \partial \bar{z}_{j}} d z_{i} \otimes d \bar{z}_{j}$.

When $D$ is strictly pseudo-convex, uniqueness and a formal approximation solution $\rho$ of $(1.2)$ with $u=-\log (-\rho)$ being plurisubharmonic in $D$ were given by Fefferman [8] earlier; the existence of such a solution was proved by Cheng and Yau [7] with $\rho \in C^{n+3 / 2}(\bar{D})$. Lee and Melrose [22] gave an asymptotic expansion solution for $\rho$. In particular, they proved that $\rho \in C^{n+2-\epsilon}(\bar{D})$ for any $\epsilon>0$. In general, $\rho$ fails to be in $C^{n+2}(\bar{D})$. Further informations about complex Monge-Ampère equations and pseudoHermitian manifolds can be found (for examples) in: [1-6, 11-17, 23, 26-35] and [38].

When $M=\left\{z \in \mathbb{C}^{n+1}: \rho(z)=0\right\}$ is hypersurface in $\mathbb{C}^{n+1}$, using the notation of $J(\rho)$, the following formula for the Webster pseudo-Ricci curvature of $(M, \theta)$ was given by $\mathrm{Li}$ and Luk in [30]:

$$
\operatorname{Ric}_{z}(w, \bar{v})=-L_{\log J(\rho)(z)}(w, \bar{v})+(n+1) \frac{\operatorname{det} H(\rho)(z)}{J(\rho)(z)} L_{\rho(z)}(w, \bar{v})
$$

for $v, w \in H_{z}(M)$, holomorphic tangent space of $M$ at $z$, where

$$
L_{u(z)}(w, \bar{v})=\sum_{j, k=1}^{n+1} \frac{\partial^{2} u(z)}{\partial z_{j} \partial \bar{z}_{k}} w_{j} \bar{v}_{k} .
$$


Assume that $\phi: D \rightarrow B_{n+1}$ is a biholomorphic mapping, and if $\rho(z)=$ $|\phi(z)|^{2}-1$, then $\operatorname{det} H(\rho)=J(\rho)=\left|\operatorname{det} \phi^{\prime}(z)\right|^{2}$ on $D$ and $\log J(\rho)$ is pluriharmonic in $D$. By (1.4), we have $R_{\theta}=n(n+1)$ on $\partial D$.

The main purpose of the current paper is to prove the converse is also true, we state it as the following theorem.

Theorem 1.1. Let $\rho \in C^{3}(\bar{D})$ be a defining function for $D \subset \mathbb{C}^{n+1}$ so that $u(z)=-\log (-\rho(z))$ is strictly plurisubharmonic in $D$. Let $M=\partial D$ and $\theta=$ $\frac{1}{2 i}(\partial \rho-\bar{\partial} \rho)$. Assume $\log J(\rho)$ is harmonic in the Kähler metric $\frac{\partial^{2} u}{\partial z_{i} \partial \bar{z}_{j}} d z_{i} \otimes$ $d \bar{z}_{j}$, we have the following two statements hold:

(a) If $R_{\theta} \equiv c>0$, constant on $\partial D$, then $D$ is biholomorphic to the unit ball $B_{n+1}$ in $\mathbb{C}^{n+1}$.

(b) Webster pseudo-scalar curvature

$$
R_{\theta}=n(n+1) \frac{\operatorname{det} H(\rho)}{J(\rho)} \text { on } \partial D \text {. }
$$

Notice that if $u(z)=-\log (-\rho(z))$ is the potential function for the Kähler-Einstein metric for $D$, then $J(\rho)=1$ on $D$. By Theorem 1.1 and Theorem 3.1 in [24], we have the following corollary.

Corollary 1.2. Let $D$ be a smoothly bounded strictly pseudo-convex domain in $\mathbb{C}^{n+1}$. Assume that $u(z)=-\log (-\rho(z))$ is the potential function for the Kähler-Einstein metric for $D$ (defined by (1.3) with $f \equiv 1)$ and $\theta=\frac{1}{2 i}(\partial \rho-$ $\bar{\partial} \rho)$ on $M=\partial D$. If $R_{\theta} \equiv c>0$, constant on $\partial D$, then there is a biholomorphic map $\phi: D \rightarrow B_{n+1}$ so that $\operatorname{det} \phi^{\prime}(z)$ is a constant on $D$.

The paper is organized as follows: In Section 2, we provide several main lemmas. In Section 3, we will prove part (b) of Theorem 1.1. Finally, the proofs of Part(a) of Theorem 1.1 and Corollary 1.2 are given in Section 4.

\section{Main lemmas}

Let $D=\left\{z \in \mathbb{C}^{n}: \rho(z)<0\right\}$ with $C^{2}$ defining function $\rho$. Let $u(z)=-\log$ $(-\rho(z))$ be plurisubharmonic in $D$. Then the relation between $J(\rho)$ and det $H(u)$ is given by the following lemma.

Lemma 2.1. Let $u \in C^{2}(D)$ and let $\rho(z)=-e^{-u}$. Then

$$
\operatorname{det} H(u)=J(\rho) e^{(n+1) u} .
$$


Proof. By viewing $\bar{\partial} u$ as a 1 by $n$ matrix and $(\bar{\partial} u)^{*}$ is its adjoint matrix, one has that

$$
\bar{\partial} \rho=-\rho \bar{\partial} u, \quad H(\rho)(z)=-\rho(z) H(u)+\rho(z)(\bar{\partial} u)^{*} \bar{\partial} u
$$

Thus

$$
\begin{aligned}
J(\rho) & =-\operatorname{det}\left[\begin{array}{cc}
\rho & -\rho \bar{\partial} u \\
-\rho(\bar{\partial} u)^{*} & -\rho H(u)+\rho(\bar{\partial} u)^{*} \bar{\partial} u
\end{array}\right] \\
& =-(\rho)^{n+1} \operatorname{det}\left[\begin{array}{cc}
1 & -\bar{\partial} u \\
-(\bar{\partial} u)^{*} & -H(u)+(\bar{\partial} u)^{*} \bar{\partial} u
\end{array}\right] \\
& =-(\rho)^{n+1} \operatorname{det}\left[\begin{array}{cc}
1 & -\bar{\partial} u \\
0 & -H(u)+(\bar{\partial} u)^{*} \bar{\partial} u-(\bar{\partial} u)^{*} \bar{\partial} u
\end{array}\right] \\
& =-(\rho)^{n+1} \operatorname{det}(-H(u)) \\
& =e^{-(n+1) u} \operatorname{det} H(u) .
\end{aligned}
$$

Therefore, (2.1) holds and the lemma is proved.

Assume that $u$ is strictly plurisubharmonic in $D$, we let $\left[u^{i \bar{j}}\right]^{t}=H(u)^{-1}$, and use the following notations:

$$
u_{i \bar{j}}=\frac{\partial^{2} u}{\partial z_{i} \partial \bar{z}_{j}}, \quad u_{i}=\frac{\partial u}{\partial z_{i}}, \quad u_{\bar{j}}=\frac{\partial u}{\partial \bar{z}_{j}}
$$

and

$$
|\partial u|_{u}^{2}:=u^{i \bar{j}} \partial_{i} u \partial_{\bar{j}} u, \quad T(z):=|\partial u|_{u}^{2}+e^{-u}
$$

Then if $\rho(z)=-e^{-u(z)}$, then by Lemma 2.2 in [24],

$$
\operatorname{det} H(\rho)=e^{-n u} \operatorname{det} H(u)\left(1-|\partial u|_{u}^{2}\right)=J(\rho) e^{u}\left(1-|\partial u|_{u}^{2}\right) .
$$

Since $u$ is strictly plurisubharmonic in $D$, by (2.1), we have $J(\rho)>0$ on $D$. Thus

$$
\frac{\operatorname{det} H(\rho)}{J(\rho)}-1=e^{u}(1-T(z)) .
$$

Then the following lemma was included in the proof of part(b) of Theorem 2.4 in [24], given in pages 468-470. 
Lemma 2.2. Let $D$ be a bounded pseudo-convex domain in $\mathbb{C}^{n}$. Let $u \in$ $C^{4}(D)$ be strictly plurisubharmonic in $D$, and let $\rho(z)=-e^{-u}$. If $-\log J(\rho)$ is subharmonic in the metric $u_{i j} d z_{i} \otimes d \bar{z}_{j}$, then

$$
\sum_{i, j=1}^{n} u^{i \bar{j}} \partial_{i \bar{j}}\left(-\frac{\operatorname{det} H(\rho)}{J(\rho)}\right)(z) \geq 0, \quad z \in D
$$

The next result is the main theorem of this section.

Theorem 2.3. Let $\rho \in C^{3}(\bar{D})$ be a defining function for $D \subset \mathbb{C}^{n}$ so that $u(z)=-\log (-\rho(z))$ is strictly plurisubharmonic in D. If $-\log J(\rho)$ is subharmonic in Kähler metric $u_{i \bar{j}} d z_{i} \otimes d \bar{z}_{j}$, and if there is a positive constant $c$ so that

$$
\frac{\operatorname{det} H(\rho)}{J(\rho)} \equiv c \quad \text { on } \quad \partial D
$$

then

$$
\frac{\operatorname{det} H(\rho)}{J(\rho)} \equiv c \text { on } D .
$$

Proof. Since $\operatorname{det} H(\rho) / J(\rho) \equiv c>0$ on $\partial D$, replacing $\rho$ by $c \rho$ if it is necessary, without loss of generality, we may assume that $c=1$. We can write

$$
\frac{\operatorname{det} H(\rho)}{J(\rho)}:=1+A(z) \rho(z), \quad z \in D
$$

Since $-\log J(\rho)$ is subharmonic in the metric $u_{i \bar{j}} d z_{i} \otimes d \bar{z}_{j}$, by Lemma 2.2, we have that $\operatorname{det} H(\rho) / J(\rho)$ attains its minimum over $\bar{D}$ at some point in $\partial D$. Thus (2.10) holds with $A(z) \leq 0$ in $D$. By (2.6), one has

$$
1-T(z)=\frac{\operatorname{det} H(\rho)}{J(\rho)} e^{-u}-e^{-u}=(1+A(z) \rho)(-\rho)+\rho .
$$

Thus, by (2.10),

$$
T(z)=1-\rho(z)+(1+A \rho) \rho=1+A(z) \rho(z)^{2} .
$$

We claim that $T(z) \equiv 1$ on $D$ (i.e., $\operatorname{det} H(\rho) / J(\rho) \equiv 1$ on $D)$.

By Lemma 2.2, if there is $z_{0} \in D$ so that $\operatorname{det} H(\rho)\left(z_{0}\right) / J(\rho)\left(z_{0}\right)=1$, then $\operatorname{det} H(\rho) / J(\rho) \equiv 1$ on $D$ (i.e., $A \equiv 0)$. Otherwise, we have $A(z)<0$ on $D$, we will prove there is a contradiction. 
Let $C=\max \{-A(z): z \in \bar{D}\}$. Then $C \in(0, \infty)$, and by $(2.12)$, we have

$$
0<(1-T) \leq C \rho(z)^{2}=C e^{-2 u}, \quad \text { on } D
$$

By (2.12) again, one has $T(z):=|\partial u|_{u}^{2}+e^{-u}<1$ on $D$. Since $e^{-u}=0$ on $\partial D$ and $e^{-u}>0$ in $D$, one can easily see that

$$
b:=\max \left\{e^{-u(z)}: z \in D\right\}<1, \quad z \in D
$$

By (2.13), we have

$$
(1-T)^{-3 / 4} \geq C^{-3 / 4} e^{3 u / 2}, \quad \frac{1}{1-e^{u(z)}} \leq \frac{1}{1-b} .
$$

Note: This is a key place we use the condition $\operatorname{det} H(\rho) / J(\rho)=1$ on $\partial D$.

For any fixed positive integer $m$ satisfying

$$
m \geq C^{3 / 4} \frac{(n+1)^{2}}{(1-b)^{2}}
$$

it is easy to see that

$$
m(1-T)^{-3 / 4}-\frac{(1+n)}{\left(1-e^{-u}\right)}-\frac{(1+n)^{2}|\partial u|^{2}}{4\left(1-e^{-u}\right)^{2}} e^{u} \geq 0, \quad z \in D .
$$

Let

$$
\mathcal{L}_{n}:=u^{i \bar{j}} \frac{\partial^{2}}{\partial z_{i} \partial \bar{z}_{j}}-n \operatorname{Re}\left(u^{i \bar{j}_{i}} u_{i} \partial_{\bar{j}}\right)
$$

and

$$
\mathcal{L}_{m, n}=\mathcal{L}_{n}+m(1-T)^{-3 / 4} \operatorname{Re}\left(\partial_{i} T u^{i \bar{j}} \partial_{\bar{j}}\right)
$$

For any $z_{0} \in D$, we will show $\mathcal{L}_{m, n} T\left(z_{0}\right) \geq 0$. By a holomorphic change of coordinates, without loss of generality, we may assume that

$$
u_{i \bar{j} k}\left(z_{0}\right)=0, \quad 1 \leq i, j, k \leq n
$$

It was proved by the author in [24] that if $T(z) \leq 1$ on $D$ and if $u$ is the strictly plurisubharmonic solution for the Monge-Ampère equation:

$$
\operatorname{det} H(u)=J e^{(n+1) u} \text { in } D ; \quad u=\infty \text { on } \partial D
$$


with $-\log J$ is subharmonic in the metric $u_{i j} d z_{i} \otimes d \bar{z}_{j}$, then

$$
\begin{aligned}
u^{i \bar{j}} \partial_{i \bar{j}} T\left(z_{0}\right) & =u^{i \bar{j}}\left(u^{k \bar{\ell}} u_{k} u_{\bar{\ell}}+e^{-u}\right) \\
& \geq n(1-T)-|\partial u|_{u}^{2}\left(1-e^{-u}\right)+u^{i \bar{j}} u^{k \bar{\ell}} u_{i k} u_{\overline{j \ell}} .
\end{aligned}
$$

Note: This is (2.24) in [24] while (2.21) in [24] becomes inequality with our assumption $u^{i \bar{j}} \partial_{i \bar{j}} \log J(\rho)\left(z_{0}\right) \leq 0$.

By (2.20), one has that $\partial_{k} u^{i \bar{j}}\left(z_{0}\right)=0$. Thus, for $1 \leq j \leq n$, one has

$$
\partial_{\bar{j}} T\left(z_{0}\right)=u^{k \bar{\ell}} u_{k} u_{\overline{j \ell}}+u_{\bar{j}}\left(1-e^{-u}\right), \quad \partial_{i} T\left(z_{0}\right)=u^{k \bar{\ell}} u_{i k} u_{\bar{j}}+u_{i}\left(1-e^{u}\right) .
$$

Thus,

$$
u^{i \bar{j}} u_{i} \partial_{\bar{j}} T\left(z_{0}\right)=u^{i \bar{j}} u_{i} u^{k \bar{\ell}} u_{k} u_{\overline{j \ell}}+|\partial u|_{u}^{2}\left(1-e^{-u}\right)
$$

and

$$
u^{i \bar{j}} \partial_{i \bar{j}} T\left(z_{0}\right) \geq n(1-T)-u^{i \bar{j}} u_{i} \partial_{\bar{j}} T+u^{i \bar{j}} u_{i} u^{k \bar{\ell}} u_{k} u_{\overline{j \ell}}+u^{i \bar{j}} u^{k \bar{\ell}} u_{i k} u_{\overline{j \ell}} .
$$

Thus,

$$
\mathcal{L}_{n} T \geq n(1-T)-\operatorname{Re}(1+n) u^{i \bar{j}} u_{i} \partial_{\bar{j}} T+\operatorname{Re} u^{i \bar{j}} u_{i} u^{k \bar{\ell}} u_{k} u_{\overline{j \ell}}+u^{i \bar{j}} u^{k \bar{\ell}} u_{i k} u_{\overline{j \ell}} .
$$

By (2.23), one has

$$
u_{i}\left(z_{0}\right)=\frac{1}{1-e^{-u}}\left[\partial_{i} T\left(z_{0}\right)-u^{k \bar{\ell}} u_{\bar{\ell}} u_{i k}\left(z_{0}\right)\right]
$$

Thus,

$$
\begin{aligned}
- & (1+n) \operatorname{Re} u^{i \bar{j}} u_{i} \partial_{\bar{j}} T \\
& =\frac{1+n}{1-e^{-u}}\left[-u^{i \bar{j}} \partial_{i} T \partial_{\bar{j}} T+u^{i \bar{j}} u^{k \bar{\ell}} u_{\bar{\ell}} u_{i k} \partial_{\bar{j}} T\right] \\
& \geq-\left[\frac{(1+n)}{\left(1-e^{-u}\right)}+\frac{(1+n)^{2}|\partial u|^{2}}{4\left(1-e^{-u}\right)^{2}} e^{u}\right] u^{i \bar{j}} \partial_{i} T \partial_{\bar{j}} T-e^{-u} u^{i \bar{j}} u^{k \bar{\ell}} u_{i k} u_{\bar{j} \bar{\ell}}
\end{aligned}
$$

and

$$
\operatorname{Re} u^{i \bar{j}} u_{i} u^{k \bar{\ell}} u_{k} u_{\overline{j \ell}}\left(z_{0}\right) \geq-|\partial u|_{u}^{2} u^{i \bar{j}} u^{k \bar{\ell}} u_{i k} u_{\overline{j \ell}}\left(z_{0}\right)
$$


Combining (2.26), (2.28) and (2.29), one has

$$
\begin{aligned}
\mathcal{L}_{n} T\left(z_{0}\right) \geq & n(1-T)+\left(1-|\partial u|^{2}-e^{-u}\right) u^{i \bar{j}} u^{k \bar{\ell}} u_{i k} u_{\overline{j \ell}} \\
& -\left[\frac{(1+n)}{\left(1-e^{-u}\right)}+\frac{(1+n)^{2}|\partial u|^{2}}{4\left(1-e^{-u}\right)^{2}} e^{u}\right] u^{i \bar{j}} \partial_{i} T \partial_{\bar{j}} T .
\end{aligned}
$$

Therefore,

$$
\begin{aligned}
\mathcal{L}_{m, n} T\left(z_{0}\right) \geq & n(1-T)+(1-T) u^{i \bar{j}} u^{k \bar{\ell}} u_{i k} u_{\overline{j \ell}} \\
& +\left[\frac{m}{(1-T)^{3 / 4}}-\frac{(1+n)}{\left(1-e^{-u}\right)}-\frac{(1+n)^{2}|\partial u|^{2}}{4\left(1-e^{-u}\right)^{2}} e^{u}\right] u^{i \bar{j}} \partial_{i} T \partial_{\bar{j}} T \\
\geq & {\left[\frac{m}{(1-T)^{3 / 4}}-\frac{(1+n)}{\left(1-e^{-u}\right)}-\frac{(1+n)^{2}|\partial u|^{2}}{4\left(1-e^{-u}\right)^{2}} e^{u}\right] u^{i \bar{j}} \partial_{i} T \partial_{\bar{j}} T } \\
\geq & 0 .
\end{aligned}
$$

Since $\operatorname{det} H(u)=J(\rho) e^{(n+1) u}$, we have $\sum_{i=1}^{n} \partial_{i}\left[J(\rho) e^{(n+1) u} u^{i \bar{j}}\right]=0$ for all $1 \leq j \leq n$. Thus,

$$
\begin{aligned}
\operatorname{Re} & \left.\sum_{i, j=1}^{n} \partial_{i}\left[e^{-n u} e^{-4 m(1-T)^{1 / 4}} J(\rho) e^{(n+1) u} u^{i \bar{j}} \partial_{\bar{j}} T\right)\right] \\
& =e^{(n+1-n) u} e^{-4 m(1-T)^{1 / 4}}\left[\mathcal{L}_{n} T\left(z_{0}\right)+m(1-T)^{-3 / 4} u^{i \bar{j}} \partial_{i} T \partial_{\bar{j}} T\right] \\
& =e^{u} e^{-4 m(1-T)^{1 / 4}} \mathcal{L}_{m, n} T .
\end{aligned}
$$

Since

$$
u^{i \bar{j}}=(-\rho)\left(\rho^{i \bar{j}}-\frac{\rho^{i} \rho^{\bar{j}}}{|\partial \rho|_{\rho}^{2}-\rho}\right), \quad \rho^{i}=\rho^{i \bar{\ell}} \rho_{\bar{\ell}},
$$

we have

$$
e^{u} \sum_{i=1}^{n} \rho_{i} u^{i \bar{j}} \partial_{\bar{j}} T=-\frac{\rho \rho^{\bar{j}}}{|\partial \rho|_{\rho}^{2}-\rho} \partial_{\bar{j}} T=0 \quad \text { on } \quad \partial D
$$


Let $|\partial \rho|_{0}^{2}=\sum_{j=1}^{n}\left|\partial_{j} \rho(z)\right|^{2}$. Then,

$$
\begin{aligned}
0 & \left.=\operatorname{Re} \sum_{i, j=1}^{n} \int_{\partial \Omega} \frac{\rho_{i}}{|\partial \rho|_{0}} e^{-n u} e^{-4 m(1-T)^{1 / 4}} J(\rho) e^{(n+1) u} u^{i \bar{j}} \partial_{\bar{j}} T\right) d \sigma(z) \\
& =\operatorname{Re} \int_{\Omega} \sum_{i, j=1}^{n} \partial_{i}\left(e^{-n u} e^{-4 m(1-T)^{1 / 4}} J(\rho) e^{(n+1) u} u^{i \bar{j}} \partial_{\bar{j}} T\right) d v \\
& =\int_{\Omega} e^{u} e^{-4 m(1-T)^{1 / 4}} \mathcal{L}_{m, n} T(z) d v(z)
\end{aligned}
$$

(2.31) shows that the integrand in the last integral is non-negative on $D$. Thus, the above identity implies that

$$
e^{u} e^{-4 m(1-T)^{1 / 4}} \mathcal{L}_{m, n} T(z)=0, \quad z \in D
$$

Therefore,

$$
\mathcal{L}_{m, n} T(z) \equiv 0, \quad \text { in } D
$$

Maximum principle (for both $T$ and $-T$ ) implies that

$$
\max \{T(z): z \in \bar{D}\}=\max \{T(z): z \in \partial D\}=1
$$

and

$$
\min \{T(z): z \in \bar{D}\}=\min \{T(z): z \in \partial D\}=1
$$

Therefore,

$$
T(z) \equiv 1, \quad \text { and } \quad \frac{\operatorname{det} H(\rho)}{J(\rho)} \equiv 1 \quad \text { in } D .
$$

This contradicts to $A(z)<0$ on $D$ (i.e., $T(z)<1$ on $D$ ). Therefore, our claim $T(z) \equiv 1$ is proved, and so

$$
\frac{\operatorname{det} H(\rho)}{J(\rho)} \equiv 1 \quad \text { in } D .
$$

Therefore, the proof of Theorem 2.3 is complete.

The following result essentially is the corollary of the main theorem of Stoll [33], Burns [3] and Wong [37] in our notation: 
Corollary 2.4. Let $\rho \in C^{3}(\bar{D})$ be a defining function for $D \subset \mathbb{C}^{n}$ so that $u(z)=-\log (-\rho(z))$ is strictly plurisubharmonic in $D$. Let $M=\partial D$ and $\theta=\frac{1}{2 i}(\partial \rho-\bar{\partial} \rho)$. If $\operatorname{det} H(\rho) / J(\rho)$ is a positive constant on the boundary $\partial D$ of $D$, then $D$ is biholomorphic to the unit ball $B_{n}$ in $\mathbb{C}^{n}$.

Proof. Since $u=-\log (-\rho)$ is plurisubharmonic in $D$, we have $J(\rho)>0$ on $D$. Notice that $\operatorname{det} H(\rho) / J(\rho)$ is a positive constant on $D$, we have $\rho$ is strictly plurisubharmonic in $D$. Let $z_{0} \in D$ be such that

$$
m=-\min \{\rho(z): z \in \bar{D}\}=-\rho\left(z_{0}\right)
$$

Then $\partial \rho\left(z_{0}\right)=0$ and

$$
J(\rho)\left(z_{0}\right)=m \operatorname{det} H(\rho)\left(z_{0}\right)
$$

Let

$$
\tau(z)=\rho(z)+m
$$

Then $\tau: D \rightarrow[0, m)$ is smooth, onto and strictly plurisubharmonic in $D$. Moreover, since $|\partial \tau|_{\tau}^{2}=|\partial \rho|_{\rho}^{2}$ and $\operatorname{det} H(\rho)=\operatorname{det} H(\tau)$, we have

$$
J[\tau]=-\operatorname{det} H(\tau)\left[\tau-|\partial \tau|_{\tau}^{2}\right]=-m \operatorname{det} H(\rho)+J(\rho)
$$

Therefore,

$$
\frac{\operatorname{det} H(\rho)}{J(\rho)} \equiv \text { constant on } D \Longleftrightarrow J(\tau) \equiv 0 \quad \text { on } D \text {. }
$$

Notice that

$$
J(\tau)=-\tau^{n+1} \operatorname{det} H(\log \tau)
$$

Thus

$$
J(\tau)=0 \Longleftrightarrow \operatorname{det} H(\log \tau)=0, \quad D \backslash \tau^{-1}(0)
$$

Combining the above relations and the main theorem of Stoll [33], Burns in [3] and Wong [37], the proof of Corollary 2.4 is complete. 


\section{Proof of part(b) of Theorem 1.1}

First let us recall some notions and a formula for the Webster pseudo Ricci curvature and pseudo-scalar curvature proved in [30]. Let $M$ be a real hypersurface in $\mathbb{C}^{n+1}$ with a defining function $\rho \in C^{3}\left(\mathbb{C}^{n+1}\right)$. Let $D=$ $\left\{z \in \mathbb{C}^{n+1}: \rho(z)<0\right\}$ and let $U$ be a neighborhood of $M$. Assume that $u(z)=-\log (-\rho(z))$ is strictly plurisubharmonic in $D \cap U$. From now on, we always use $\rho$ to denote a defining function for $M$ and $\theta=\frac{1}{2 i}(\partial \rho-\bar{\partial} \rho)$ is the pseudo-Hermitian form for $M$ associated to the defining function $\rho$. We define a second-order differential operator $\mathcal{D}_{\alpha \bar{\beta}}$ associated to $\rho$ for $1 \leq \alpha, \beta \leq n$ as follows:

$$
\mathcal{D}_{\alpha \bar{\beta}}=\frac{\partial^{2}}{\partial z_{\alpha} \partial \bar{z}_{\bar{\beta}}}-\frac{\rho_{\alpha}}{\rho_{n+1}} \frac{\partial^{2}}{\partial z_{n+1} \partial \bar{z}_{\beta}}-\frac{\rho_{\bar{\beta}}}{\rho_{\overline{n+1}}} \frac{\partial^{2}}{\partial z_{\alpha} \partial \bar{z}_{n+1}}+\frac{\rho_{\alpha} \rho_{\bar{\beta}}}{\left|\rho_{n+1}\right|^{2}} \frac{\partial^{2}}{\partial z_{n+1} \partial \bar{z}_{n+1}} .
$$

The following explicit formula for the Webster pseudo-Ricci curvature and pseudo-scalar curvature in terms of defining function $\rho$ for $(M, \theta)$ was proved in $[30]$ :

Theorem 3.1. Let $M=\partial D$ be a strictly pseudo-convex hypersurface in $\mathbb{C}^{n+1}$. Let $\rho \in C^{3}(\bar{D} \cap U) \cap C^{\infty}(D \cap U)$ be a defining function for $M$ with that $J(\rho)>0$ on $\bar{D} \cap U$ and $u(z)=-\log (-\rho)$ is plurisubharmonic in $D \cap U$. Let $\theta=(\partial \rho-\bar{\partial} \rho) /(2 i)$. Then for $v, w \in H(M)=T_{1,0}(M)$, we have:

$$
\operatorname{Ric}(w, \bar{v})=-L_{\log J(\rho)}(w, \bar{v})+(n+1) \frac{\operatorname{det} H(\rho)}{J(\rho)} L_{\rho}(w, \bar{v}),
$$

where $L_{g}(w, \bar{v})=\sum_{k, j=1}^{n+1} g_{k \bar{j}}(z) w_{k} \bar{v}_{j}$ is the Levi form associated to $g$.

In a local coordinates, at those $z \in M_{1}=\left\{z \in M: \rho_{n+1}(z) \neq 0\right\}$, we have the Webster pseudo-scalar curvature

$$
R_{\theta}(z)=-\sum_{\alpha, \beta=1}^{n} h^{\alpha \bar{\beta}} \mathcal{D}_{\alpha \bar{\beta}} \log J(\rho)+n(n+1) \frac{\operatorname{det} H(\rho)}{J(\rho)},
$$

where the pseudo-Hermitian metric $h_{\alpha \bar{\beta}}=\mathcal{D}_{\alpha \bar{\beta}}(\rho)$ and $\left[h^{\alpha \bar{\beta}}\right]=\left(\left[h_{\alpha \bar{\beta}}\right]^{t}\right)^{-1}$.

Proposition 3.2. With the notation above, $h_{\alpha \bar{\beta}}=\mathcal{D}_{\alpha \bar{\beta}}(\rho)$, we have

$$
h^{\alpha \bar{\beta}}(z)=\rho^{\alpha \bar{\beta}}-\frac{\rho^{\alpha} \rho^{\bar{\beta}}}{|\partial \rho|_{\rho}^{2}}, \quad z \in M_{1}, \quad 1 \leq \alpha, \beta \leq n
$$


Proof. Since, for $1 \leq i, k \leq n$ with $h_{k \bar{j}}=\mathcal{D}_{k \bar{j}}(\rho)$

$$
\begin{aligned}
& \sum_{j=1}^{n}\left(\rho^{i \bar{j}}-\frac{\rho^{i} \rho^{\bar{j}}}{|\partial \rho|_{\rho}^{2}}\right) h_{k \bar{j}} \\
& =\sum_{j=1}^{n}\left(\rho^{i \bar{j}}-\frac{\rho^{i} \rho^{\bar{j}}}{|\partial \rho|_{\rho}^{2}}\right)\left(\rho_{k \bar{j}}-\frac{\rho_{k}}{\rho_{n+1}} \rho_{n+1 \bar{j}}-\frac{\rho_{\bar{j}}}{\rho_{\overline{n+1}}} \rho_{k \overline{n+1}}+\frac{\rho_{k} \rho_{\bar{j}}}{\left|\rho_{n+1}\right|^{2}} \rho_{n+1} \overline{n+1}\right) \\
& =\sum_{j=1}^{n} \rho^{i \bar{j}}\left(\rho_{k \bar{j}}-\frac{\rho_{k}}{\rho_{n+1}} \rho_{n+1 \bar{j}}-\frac{\rho_{\bar{j}}}{\rho_{\overline{n+1}}} \rho_{k \overline{n+1}}+\frac{\rho_{k} \rho_{\bar{j}}}{\left|\rho_{n+1}\right|^{2}} \rho_{n+1} \overline{n+1}\right) \\
& -\sum_{j=1}^{n} \frac{\rho^{i} \rho^{\bar{j}}}{|\partial \rho|_{\rho}^{2}}\left(\rho_{k \bar{j}}-\frac{\rho_{k}}{\rho_{n+1}} \rho_{n+1 \bar{j}}-\frac{\rho_{\bar{j}}}{\rho_{\overline{n+1}}} \rho_{k \overline{n+1}}+\frac{\rho_{k} \rho_{\bar{j}}}{\left|\rho_{n+1}\right|^{2}} \rho_{n+1} \overline{n+1}\right) \\
& =\delta_{i k}-\rho^{i \overline{n+1}} \rho_{k \overline{n+1}}+\frac{\rho_{k}}{\rho_{n+1}} \rho^{i \overline{n+1}} \rho_{n+1} \overline{n+1}-\frac{\rho^{i}}{\rho_{\overline{n+1}}} \rho_{k \overline{n+1}}+\rho^{i \overline{n+1}} \rho_{k \overline{n+1}} \\
& +\frac{\rho^{i} \rho_{k}}{\left|\rho_{n+1}\right|^{2}} \rho_{n+1} \overline{n+1}-\rho^{i \overline{n+1}} \frac{\rho_{k}}{\rho_{n+1}} \rho_{n+1} \overline{n+1} \\
& -\frac{\rho^{i} \rho_{k}}{|\partial \rho|_{\rho}^{2}}+\frac{\rho^{i} \rho^{\overline{n+1}}}{|\partial \rho|_{\rho}^{2}} \rho_{k \overline{n+1}}+\frac{\rho^{i} \rho_{n+1}}{|\partial \rho|_{\rho}^{2}} \frac{\rho_{k}}{\rho_{n+1}}-\frac{\rho^{i} \rho^{\overline{n+1}}}{|\partial \rho|_{\rho}^{2}} \frac{\rho_{k}}{\rho_{n+1}} \rho_{n+1} \overline{n+1} \\
& +\frac{\rho^{i}}{\rho_{\overline{n+1}}} \rho_{k \overline{n+1}}-\frac{\rho^{i} \rho^{\overline{n+1}}}{|\partial \rho|_{\rho}^{2}} \rho_{k n+1}-\frac{\rho^{i} \rho_{k}}{\left|\rho_{n+1}\right|^{2}} \rho_{n+1} \overline{n+1}+\frac{\rho^{i} \rho^{\overline{n+1}}}{|\partial \rho|_{\rho}^{2}} \frac{\rho_{k}}{\rho_{n+1}} \rho_{n+1} \overline{n+1} \\
& =\delta_{i k} \text {. }
\end{aligned}
$$

Therefore, (3.4) holds, and the proof of the proposition is complete.

Let $\Delta_{u}$ be the Beltrami-Laplacian with respect to the metric $u_{i \bar{j}} d z_{i} \otimes$ $d \bar{z}_{j}$. Then

$$
\Delta_{u}=\sum_{i, j=1}^{n+1} u^{i \bar{j}} \frac{\partial^{2}}{\partial z_{i} \partial \bar{z}_{j}}
$$

Since $u=-\log (-\rho)$, we have $($ see $[7,24])$ that

$$
u_{i \bar{j}}=\frac{\rho_{i \bar{j}}}{-\rho}+\frac{\rho_{i} \rho_{\bar{j}}}{\rho^{2}}, \quad \text { and } \quad u^{i \bar{j}}=(-\rho)\left(\rho^{i \bar{j}}-\frac{\rho^{i} \rho^{\bar{j}}}{-\rho+|\partial \rho|_{\rho}^{2}}\right)
$$


Then,

$$
\Delta_{u}=(-\rho) \sum_{i, j=1}^{n+1}\left(\rho^{i \bar{j}}-\frac{\rho^{i} \rho^{\bar{j}}}{-\rho+|\partial \rho|_{\rho}^{2}}\right) \frac{\partial^{2}}{\partial z_{i} \partial \bar{z}_{j}} .
$$

Thus if $f$ is harmonic in the metric $u_{i \bar{j}} d z_{i} \otimes d \bar{z}_{j}$, then

$$
\Delta_{u} f=\sum_{i, j=1}^{n+1}\left(\rho^{i \bar{j}}-\frac{\rho^{i} \rho^{\bar{j}}}{-\rho+|\partial \rho|_{\rho}^{2}}\right) \frac{\partial^{2} f}{\partial z_{i} \partial \bar{z}_{j}}=0
$$

Proposition 3.3. With the notation above, for any $f \in C^{2}(D)$, we have

$$
\sum_{i, j=1}^{n+1}\left(\rho^{i \bar{j}}-\frac{\rho^{i} \rho^{\bar{j}}}{|\partial \rho|_{\rho}^{2}}\right) f_{i \bar{j}}=\sum_{i, j=1}^{n}\left(\rho^{i \bar{j}}-\frac{\rho^{i} \rho^{\bar{j}}}{|\partial \rho|_{\rho}^{2}}\right) \mathcal{D}_{i \bar{j}}(f)
$$

Proof. Since

$$
\begin{aligned}
\sum_{i, j=1}^{n} & \left(\rho^{i \bar{j}}-\frac{\rho^{i} \rho^{\bar{j}}}{|\partial \rho|_{\rho}^{2}}\right) f_{i \bar{j}}-\sum_{i, j=1}^{n}\left(\rho^{i \bar{j}}-\frac{\rho^{i} \rho^{\bar{j}}}{|\partial \rho|_{\rho}^{2}}\right) \mathcal{D}_{i \bar{j}}(f) \\
= & \sum_{i, j=1}^{n}\left(\rho^{i \bar{j}}-\frac{\rho^{i} \rho^{\bar{j}}}{|\partial \rho|_{\rho}^{2}}\right)\left(\frac{\rho_{i}}{\rho_{n+1}} f_{n+1 \bar{j}}+\frac{\rho_{\bar{j}}}{\rho_{\overline{n+1}}} f_{i \overline{n+1}}-\frac{\rho_{i} \rho_{\bar{j}}}{\left|\rho_{n+1}\right|^{2}} f_{n+1} \overline{n+1}\right) \\
= & \sum_{j=1}^{n} \frac{\rho^{\bar{j}}}{\rho_{n+1}} f_{n+1 \bar{j}}-\sum_{j=1}^{n} \rho^{n+1 \bar{j}} f_{n+1 \bar{j}}+\sum_{i=1}^{n} \frac{\rho^{i}}{\rho_{\overline{n+1}}} f_{i \overline{n+1}}-\sum_{i=1}^{n} \rho^{i \overline{n+1}} f_{i \overline{n+1}} \\
& +\sum_{i=1}^{n}\left(-\frac{\rho_{i} \rho^{i}}{\left|\rho_{n+1}\right|^{2}}+\rho^{i \overline{n+1}} \frac{\rho_{i}}{\rho_{n+1}}\right) f_{n+1} \overline{n+1} \\
& -\frac{\sum_{i=1}^{n} \rho^{i} \rho_{i}}{|\partial \rho|_{\rho}^{2}} \sum_{j=1}^{n} \frac{\rho^{\bar{j}}}{\rho_{n+1}} f_{n+1 \bar{j}}-\frac{\sum_{j=1}^{n} \rho^{j} \rho_{\bar{j}}}{|\partial \rho|_{\rho}^{2}} \sum_{i=1}^{n} \frac{\rho^{i}}{\rho_{\overline{n+1}}} f_{i \overline{n+1}} \\
& +\frac{\left(\sum_{i=1}^{n} \rho^{i} \rho_{i}\right)\left(\sum_{j=1}^{n} \rho^{\bar{j}} \rho_{\bar{j}}\right)}{|\partial \rho|_{\rho}^{2}\left|\rho_{n+1}\right|^{2}} f_{n+1 \overline{n+1}}
\end{aligned}
$$




$$
\begin{aligned}
= & \left(1-\frac{\sum_{i=1}^{n} \rho^{i} \rho_{i}}{|\partial \rho|_{\rho}^{2}}\right) \sum_{j=1}^{n} \frac{\rho^{\bar{j}}}{\rho_{n+1}} f_{n+1 \bar{j}}-\sum_{j=1}^{n} \rho^{n+1 \bar{j}} f_{n+1 \bar{j}} \\
& +\left(1-\frac{\sum_{j=1}^{n} \rho^{\bar{j}} \rho_{\bar{j}}}{|\partial \rho|_{\rho}^{2}}\right) \sum_{i=1}^{n} \frac{\rho^{i}}{\rho_{\overline{n+1}}} f_{i \overline{n+1}}-\sum_{i=1}^{n} \rho^{i \overline{n+1}} f_{i \overline{n+1}}+\left(-\frac{\sum_{i=1}^{n} \rho_{i} \rho^{i}}{\left|\rho_{n+1}\right|^{2}}\right. \\
& \left.+\frac{\rho^{\overline{n+1}}}{\rho_{n+1}}-\rho^{n+1} \overline{n+1}+\frac{\left(\sum_{i=1}^{n} \rho^{i} \rho_{i}\right)\left(\sum_{j=1}^{n} \rho^{\bar{j}} \rho_{\bar{j}}\right)}{|\partial \rho|_{\rho}^{2}\left|\rho_{n+1}\right|^{2}}\right) f_{n+1} \overline{n+1} \\
= & -\sum_{j=1}^{n}\left[\rho^{n+1 \bar{j}}-\frac{\rho^{n+1} \rho^{j}}{|\partial \rho|_{\rho}^{2}}\right] f_{n+1}-\sum_{i=1}^{n}\left[\rho^{i n+1}-\frac{\rho^{n+1} \rho^{i}}{|\partial \rho|_{\rho}^{2}}\right] f_{i \overline{n+1}} \\
& -\left[\rho^{n+1} \overline{n+1}-\frac{\rho^{n+1} \rho^{\overline{n+1}}}{|\partial \rho|_{\rho}^{2}}\right] f_{n+1} \overline{n+1} .
\end{aligned}
$$

Moving the right side to the left hand side, we have

$$
\sum_{i, j=1}^{n+1}\left(\rho^{i \bar{j}}-\frac{\rho^{i} \rho^{\bar{j}}}{|\partial \rho|_{\rho}^{2}}\right) f_{i \bar{j}}-\sum_{i, j=1}^{n}\left(\rho^{i \bar{j}}-\frac{\rho^{i} \rho^{\bar{j}}}{|\partial \rho|_{\rho}^{2}}\right) \mathcal{D}_{i \bar{j}}(f)=0
$$

Therefore, the proof of (3.9) is complete, and so is the proof of the proposition.

Proof of part(b) of Theorem 1.1. Let $f(z)=\log J(\rho)(z)$ be harmonic in the metric $u_{i j} d z_{i} \otimes d \bar{z}_{j}$. By (3.8), and then by (3.9), we have for all $z \in M=\partial D$

$$
0=\sum_{i, j=1}^{n+1}\left(\rho^{i \bar{j}}-\frac{\rho^{i} \rho^{\bar{j}}}{|\partial \rho|_{\rho}^{2}}\right) \frac{\partial^{2} f}{\partial z_{i} \partial \bar{z}_{j}}=\sum_{i, j=1}^{n}\left(\rho^{i \bar{j}}-\frac{\rho^{i} \rho^{\bar{j}}}{|\partial \rho|_{\rho}^{2}}\right) \mathcal{D}_{i \bar{j}}(f)(z)
$$

By (3.4) and $f=\log J(\rho)$, we have

$$
\sum_{\alpha, \beta=1}^{n} h^{\alpha \bar{\beta}} \mathcal{D}_{\alpha \bar{\beta}}(\log J(\rho))=0, \quad z \in M_{1}
$$

Applying the formula (3.3) for the Webster pseudo-scalar curvature $R_{\theta}$, we have that $R_{\theta}=n(n+1) \operatorname{det} H(\rho) / J(\rho)$, i.e., (1.5) holds, and the proof of part(b) of Theorem 1.1 is complete. 


\section{Proof of Theorem 1.1 and Corollary 1.2}

We have proved part(b) of Theorem 1.1. Now we prove part(a) of Theorem 1.1.

Proof of part(a) of Theorem 1.1. Since $\log J(\rho)$ is harmonic in the metric $u_{i j} d z_{i} \otimes d \bar{z}_{j}$ in $D$ and the pseudo-scalar curvature $R_{\theta}$ is constant $c$ on $\partial D$, by part(b) of Theorem 1.1, we have

$$
c=R_{\theta}=n(n+1) \frac{\operatorname{det} H(\rho)}{J(\rho)} \quad \text { on } \partial D
$$

Therefore,

$$
\frac{\operatorname{det} H(\rho)}{J(\rho)}=\frac{c}{n(n+1)}>0 \quad \text { on } \partial D
$$

This with $-\log J(\rho)$ being harmonic in the metric $u_{i \bar{j}} d z_{i} \otimes d \bar{z}_{j}$, we have that all conditions of Theorem 2.3 hold. By Theorem 2.3, we have

$$
\frac{\operatorname{det} H(\rho)}{J(\rho)}=\frac{c}{n(n+1)}>0 \quad \text { on } D
$$

By Corollary 2.4, we have that $D$ is biholomorphic to $B_{n+1}$, and the proof of part(a) of Theorem 1.1 is complete.

Proof of Corollary 1.2. Since $u$ is the potential function for $D$, we have $J(\rho) \equiv 1$. Hence, $\log J(\rho)=0$ is harmonic in $u_{i \bar{j}} d z_{i} \otimes d \bar{z}_{j}$. By part(b) of Theorem 1.1, we have $R_{\theta}=c$ on $\partial D$, and then by part(a) of Theorem 1.1 that $D$ is biholomorphic to $B_{n+1}$. Moreover, $\operatorname{det} H(\rho) \equiv c$ on $D$. Let $\rho^{0}=c \rho$. $\operatorname{det} H\left(\rho^{0}\right) / J\left(\rho^{0}\right) \equiv 1$ on $D$. Then by $(2.37)$ and $(2.38)$, there is $z_{0} \in D$ with

$$
\rho^{0}\left(z_{0}\right)=\min \left\{\rho^{0}(z): z \in D\right\}=-\frac{\operatorname{det} H\left(\rho^{0}\right)\left(z_{0}\right)}{J\left(\rho^{0}\right)\left(z_{0}\right)}=-1
$$


By (2.6), we have $T(z) \equiv 1$ on $D$. By $(2.5)$ in [24], we have $\operatorname{det} H\left(\log \left(1+\rho^{0}\right)\right)(z)=0, \quad$ for all $z \in D$ with $\rho^{0}(z)>-1$.

Applying part(ii) of Theorem 1.2 in [24], there is a constant Jacobian biholomorphic mapping $\phi: D \rightarrow B_{n+1}$. The proof of Corollary 1.2 is complete.

\section{Acknowledgment}

A part of this work was done when the author was visiting Fujian Normal University, China.

\section{References}

[1] S.M. Baouendi, P. Ebenfelt and L.P. Rothschild, Real Submanifolds in Complex Space and their Mappings, Math Series 47, Princeton University Press, Princeton, New Jersey, 1999.

[2] R. Beals, C. Fefferman and R. Grossman, Strictly pseudo-convex domains in $\mathbb{C}^{n}$, Bull. Amer. Math. Soc. (N.S.) 8 (1983), 125-322.

[3] D. Burns, Curvatures of Monge-Ampère foliations and parabolic manifolds, Ann. Math. 115 (1982), 349-373.

[4] D. Burns Jr. and S. Shnider, Spherical hypersurfaces in complex manifolds, Invent. Math. 33 (1976), 223-246.

[5] L. Caffarelli, J.J. Kohn, L. Nirenberg and J. Spruck, The Dirichlet problem for nonlinear second-order elliptic equations, II: complex MongeAmpère, and uniformly elliptic equations, Comm. Pure Appl. Math. 38 (1985), 209-252.

[6] S.S. Chern and S. Ji, On the Riemann mapping theorem, Ann. Math. 144 (1996), 421-439.

[7] S.Y. Cheng and S.T. Yau, On the existence of a complex Kähler metric on non-compact complex manifolds and the regularity of Fefferman's equation, Comm. Pure Appl. Math. 33 (1980), 507-544.

[8] C. Fefferman, Monge-Ampère equations, the Bergman kernel, and geometry of pseudo-convex domains, Ann. Math. 103 (1976), 395-416.

[9] N. Gamara, The CR Yamabe conjecture the case $n=1$, J. Eur. Math. Soc. 3 (2001), 105-137. 
[10] N. Gamara and R. Yacoub, CR Yamabe conjecture-the conformally flat case, Pacific J. Math. 201 (2001), 121-175.

[11] C.R. Graham, Scalar boundary invariants and the Bergman type kernel, Complex Analysis, II (College Park, Maryland, 1985-85), Lecture Notes in Math. 1276, Springer, Berlin, 1987, 108-135.

[12] B. Guan, The Dirichlet problem for complex Monge-Ampère equations and Regularity of the Green's function, Comm. Anal. Geom. 6 (1998), 687-703.

[13] B. Guan and P. Guan, Convex hypersurfaces of prescribed curvature, Ann. Math. 156 (2002), 655-674.

[14] P. Guan, Extremal function associated to intrinsic norm, Ann. Math. 156 (2002), 197-211.

[15] X. Huang, On some problems in several complex variables and $C R$ geometry, First International Congress of Chinese Mathematicians, Beijing, 1998, 383-396.

[16] X. Huang and S. Ji, Global holomorphic extension of a local map and a Riemann mapping theorem for algebraic domains, Math. Res. Lett. 5 (1-2) (1998), 247-260.

[17] X. Huang, S. Ji and S.S.T. Yau, An example of a real analytic strongly pseudo-convex hypersurface which is not holomorphically equivalent to any algebraic hypersurface, Ark. Mat. 39 (2001), 75-93.

[18] D. Jerison and J. Lee, The Yamabe problem on CR manifolds, J. Diff. Geom. 25 (1987), 167-197.

[19] - Extremal for the Sobolev inequality on the Heisenberg group and the CR Yamabe problem, J. Amer. Math. Soc. 1 (1) (1988), 1-13.

[20] S.G. Krantz and S.-Y. Li, On the existence of smooth plurisubharmonic solutions for certain degenerate Monge-Ampère equations, Complex Vari. 41 (2000), 207-219.

[21] J.M. Lee, The Fefferman metric and pseudo-Hermitian invariants, Trans. Amer. Math. Soc. 296 (1) (1986), 411-429.

[22] J. Lee and R. Melrose, Boundary behavior of the complex MongeAmpère equation, Acta Math. (1982), 159-192.

[23] L. Lempert, Solving the degenerate Monge-Ampère equation with one concentrated singularity, Math. Ann. 263 (1983), 515-532. 
[24] S.-Y. Li, Characterization for balls with potential function of KählerEinstein metrics for domains in $\mathbb{C}^{n}$, Comm. Anal. Geom. 13 (2005), 461-478.

[25] - On the existence and regularity of Dirichlet problem for complex Monge-Ampère equations on weakly pseudo-convex domains, Calc. Var. PDEs 20 (2004), 119-132.

[26] - The Neumann problem for complex Monge-Ampère equation, Ind. Univ. J. Math. 44 (1995), 1099-1122.

[27] - On the Dirichlet problem for symmetric function equations of eigenvalues of complex Hessian, Asian J. Math. 8 (2004), 87-106.

[28] —, On pseudo-Hermitian CR manifolds, AMS/IP Stud. Adv. Math. 42 (2008), 467-481.

[29] S.-Y. Li and H.-S. Luk, The Sharp lower bound for the first positive eigenvalues of sub-Laplacian on the pseudo-hermitian manifold, Proc. AMS 132 (2004), 789-798.

[30] - An explicit formula for the Webster pseudo Ricci curvature on real hypersurfaces and its application for characterizing balls in $\mathbb{C}^{n}$, Comm. Anal Geom. 14 (2006), 673-701.

[31] — An explicit formula for the Webster torsion on real hypersurfaces and its application to the torsion-free hypersurfaces in $\mathbb{C}^{n}$, Sci. China 49 (2006), 1662-1682.

[32] N. Mok and S.T. Yau, Completeness of the Kähler-Einstein metric on bounded domains and the characterization of domains of holomorphy by curvature conditions, The mathematical Heritage of Henri Poincarè Part 1 (Bloomington, Ind., 1980), Proc. Symposia. Pure Math., 39, Amer. Math. Soc., Providence, RI, 1983, 41-59.

[33] W. Stoll, The characterization of strictly parabolic manifolds, Ann. Scuola Norm. Sup. Pisa Cl. Sci. (4) 7 (1) (1980), 87-154.

[34] N. Tanaka, A Differential geometric study on strongly pseudo-convex manifolds, Kinokunija Company Ltd, Tokyo, 1975.

[35] S.M. Webster, On the pseudo-conformal geometry of a Kähler manifold, Math. Z. 157 (3) (1977), 265-270.

[36] - Pseudo-hermitian geometry on a real hypersurface, J. Diff. Geom. 13 (1973), 25-41. 
[37] P.-M. Wong, Geometry of the complex homogeneous Monge-Ampre equation, Invent. Math. 67 (2) (1982), 261-274.

[38] — Stability of the Monge-Ampre foliation, Math. Ann. 263 (1) (1983), 13-29.

School of Mathematics and Computer Science

FuJIAN NORMAL UNIVERSITY

FUJIAN

CHINA

E-mail address: sli@math.uci.edu

Present AdDREsS

Department of Mathematics

University OF CALIFORNIA

IRVINE

CA $92697-3875$

USA

RECEIVED OCTOBER 2, 2007 
